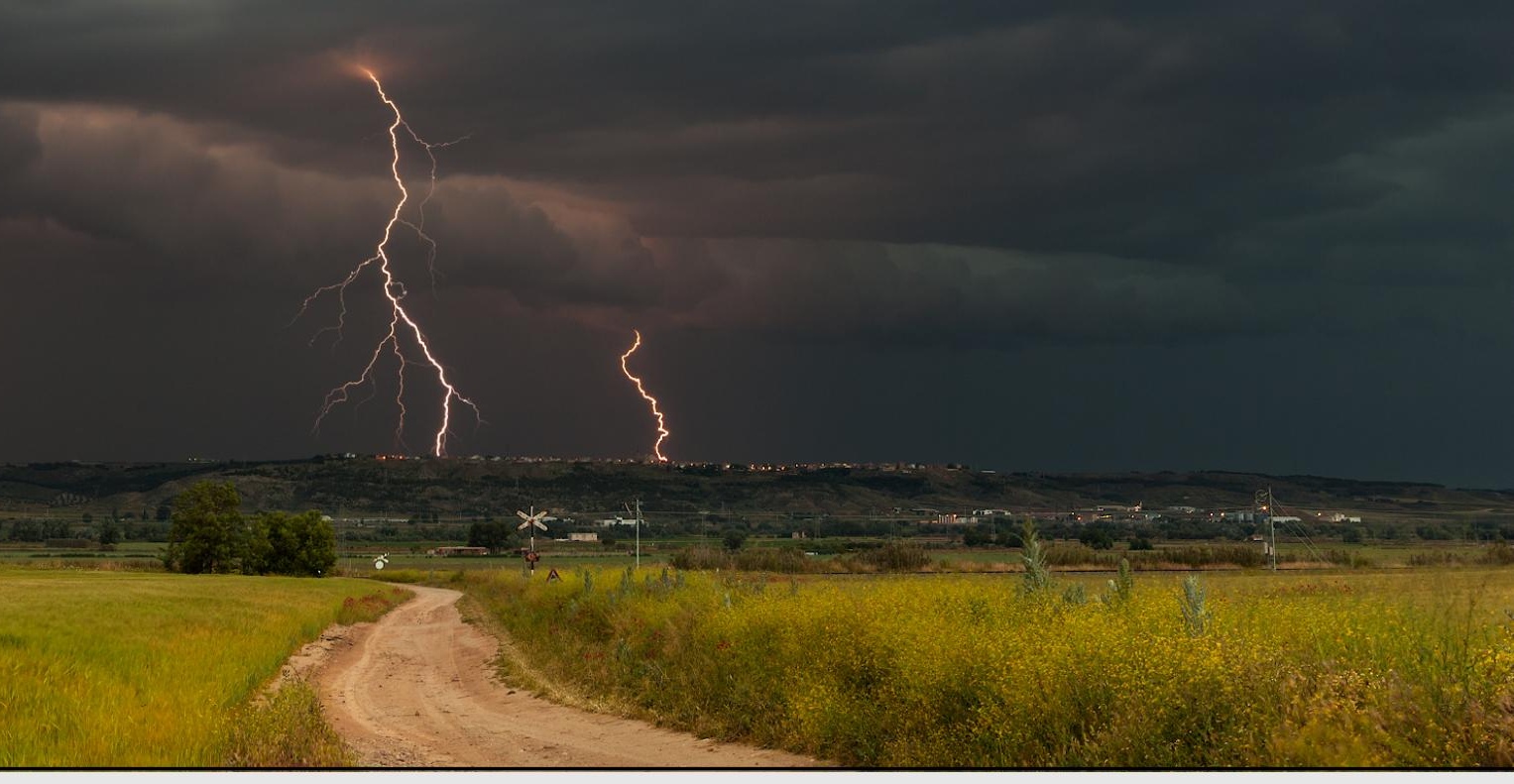

\title{
Métodos probabilistas para previsión de rayos
}

DOI: $10.31978 / 014-18-009-X .34$

\section{José AnTONio Sosa CARdo}

Delegación territorial (DT) en Andalucía, Ceuta y Melilla, Agencia Estatal de Meteorología (AEMET)

\begin{abstract}
Las tormentas eléctricas son las más extendidas y comunes, son además muy peligrosas y costosas. Se calcula que en todo momento estan descargando 2000 tormentas eléctricas sobre la superficie de la Tierra, generando unos 100 rayos por segundo. Cada día hay cerca de 45000 tormentas o casi 16 millones en un año. De éstas, 100000 azotan los EE. UU., con Florida a la cabeza de la clasificación. Su elevado número las convierte en el principal regulador del balance calorífico de la tierra. Cada año mueren 100 norteamericanos y casi 250 resultan gravementes heridos.
\end{abstract}

Las tormentas - JON ERICKSON

Los rayos son fenómenos meteorológicos adversos presentes en las tormentas. La predicción de este tipo de fenómenos con impacto en la actividad humana, es tan importante como difícil. Por un lado, los sistemas de predicción por conjuntos (SPC) aportan, en este contexto, un enfoque muy fructífero que, entre otras ventajas, permite el desarrollo de herramientas específicas. Por otro lado, la actividad eléctrica está muy relacionada con el graupel (nieve granulada) y esa relación permite aprovechar la modelización de la convección profunda y el graupel para estimar la actividad eléctrica asociada. Presentamos en este capítulo una herramienta para la previsión probabilista de rayos basada por un lado, en esa relación graupel-rayos y, por otro, en los SPC. Esta herramienta, probada experimentalmente en AEMET en predicción, con excelentes resultados.

Palabras clave: sistemas de predicción por conjuntos aplicados a la previsión de descargas eléctricas, actividad eléctrica y graupel, ensemble de hombre muy pobre, modelo HARMONIE-AROME. 
HARMONIE: Rayos estimados (rayos $/ \mathrm{km}^{2}$ ). Pas: 29-08-2017 00Z Intearacion 29-08-2017 00 - 30-08-2017 00Z

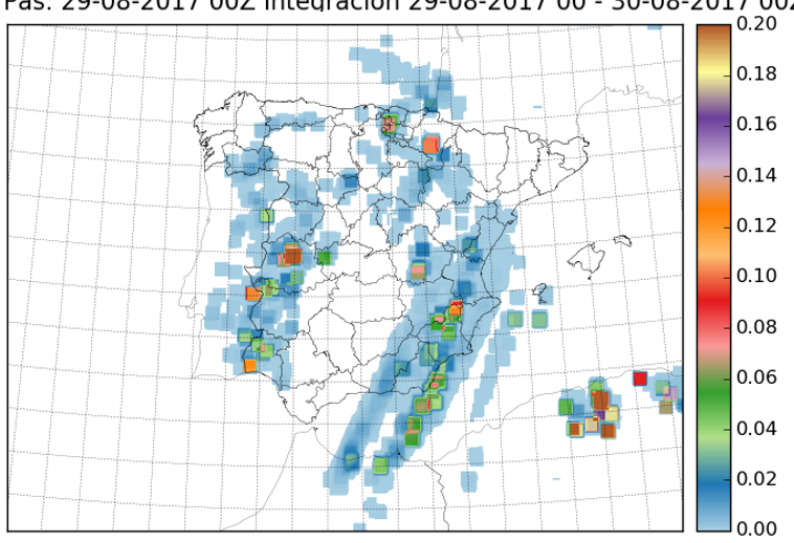

HARMONIE: Rayos estimados (rayos $/ \mathrm{km}^{2}$ ). Pas: 29-08-2017 00Z Intearacion 29-08-2017 00 - 30-08-2017 00Z

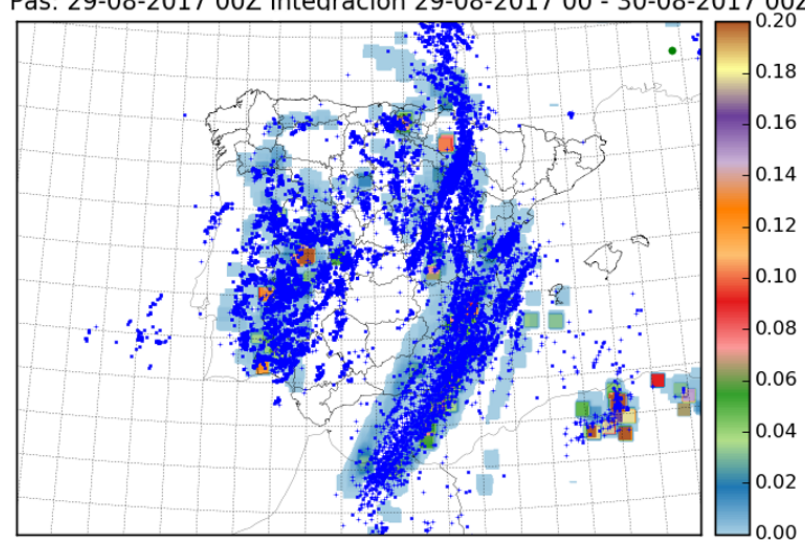

Figura 34.1: Izquierda: rayos estimados para el día 29-08-2017 (intervalo 00 UTC - 00 UTC) con la pasada de las 00 UTC del mismo día 29 de HARMONIE-AROME. Derecha: similar a la figura anterior, pero con los rayos observados en el día 29-08-2017 (intervalo 00 UTC - OO UTC) superpuestos. El parecido de ambos patrones espaciales es notable.

\subsection{Fenómenos adversos}

Las predicciones de fenómenos meteorológicos adversos son elemento clave en todo sistema de previsiones meteorológicas. Estos fenómenos son los de más impacto social y económico, mientras que también son los más complicados para dar predicciones acertadas con la antelación necesaria. Los sistemas de predicción por conjuntos (SPC) se fraguaron por la dificultad de los modelos deterministas en dar predicciones acertadas en los casos extremos. Los fenómenos extremos no son siempre adversos y viceversa: no todo fenómeno adverso es extremo, pero hay una relación entre ambos. Los predictores del tiempo encuentran grandes ventajas usando SPC pues de forma natural pueden enfocar la predicción desde un punto de vista probabilista. Pero las ventajas no terminan ahí: pueden desarrollarse herramientas especiales, basadas en SPC, específicas para la previsión de fenómenos meteorológicos adversos. Se muestra aquí uno de los ejemplos emergentes de este tipo de herramientas: previsión probabilista de rayos basada en la relación entre actividad eléctrica y graupel, una herramienta probada experimentalmente en las unidades de predicción de Agencia Estatal de Meteorología (AEMET) con muy buenos resultados.

\subsection{Actividad eléctrica y graupel}

La actividad eléctrica en la atmósfera es ya de por sí un fenómeno que puede causar daños importantes sobre personas y bienes. Además su formación va ineludiblemente ligada a nubes convectivas del tipo cumulonimbo, que suelen llevar asociados otros fenómenos que pueden ser también dañinos, como por ejemplo granizo, lluvias torrenciales, frentes de racha, tornados o fuertes corrientes verticales.

Los modelos no hidrostáticos de predicción numérica suponen una gran mejora en la modelización de la convección profunda respecto a los modelos hidrostáticos, ya que en los últimos la convección en la atmósfera estaría inhibida (caps 10 en la página 129 y 17 en la página 257). Estos modelos no hidrostáticos suelen incorporar una microfísica más rica y sofisticada, modelizándose distintos estados del agua en la atmósfera. Uno de los estados que tiene un interés especial en el pronóstico de tormentas es el llamado granizo blando, nieve granulada o graupel, que juega un papel fundamental en uno de los principales mecanismos de separación de cargas eléctricas en la atmósfera, el mecanismo denominado «graupel-ice» [6]. Existen varios trabajos que correlacionan la presencia de graupel con la actividad eléctrica observada, de forma que puede utilizarse el graupel como un pronosticador de actividad eléctrica. $[3,5,8,9]$.

Con el objeto de dar una primera idea cualitativa de cómo se comporta el pronóstico de rayos basado en el graupel para casos reales relevantes, hemos escogido un día del pasado verano con episodios tormentosos importantes, es decir, con intensa actividad eléctrica en la atmósfera: el día 29 de agosto de 2017. Ofrecemos en la Figura 34.1 mapas de rayos previstos y rayos observados para el día en cuestión. Puede observarse que los patrones espaciales previstos, sin ser 
exactamente iguales a los observados, dan una muy buena aproximación.

\subsection{Un sólo modelo: ensemble de hombre muy pobre}

Cuando no es posible contar con un ensemble completo con un número de miembros suficiente y que explore las distintas fuentes de error en la modelización atmosférica, generalmente la forma de abordar la predicción probabilista es utilizar lo que se denomina ensemble del hombre pobre (poor man's ensemble o, abreviadamente, PME, [2,7]). Es un ensemble construido con las salidas de distintos modelos de predicción numérica. Esta aproximación explora tanto las posibles desviaciones en el pronóstico debidas a incertidumbres en las condiciones iniciales como las debidas a distintas implementaciones de la física en cada uno de los modelos. La principal desventaja suele ser el número limitado de miembros disponibles y, a menudo, la heterogeneidad en su dominio, naturaleza y parámetros.

En el caso de modelos no hidrostáticos, que suelen necesitar una gran cantidad de cálculo, el área a modelizar se reduce espacialmente para ser capaces de ejecutar la pasada a tiempo, siendo bastante probable que encontremos que el área de interés la cubre sólo un modelo, lo que no permite ni siquiera construir un PME, lo que nos obliga a intentar formar un ensemble desde otra perspectiva.

A corto plazo se pueden utilizar las pasadas ejecutadas con anterioridad de un mismo modelo, para nuestra área de interés, lo que nos permite construir un pequeño ensemble con las pasadas anteriores de nuestro modelo que cubren temporalmente la ventana de pronóstico de muy corto plazo, lo que se denomina Time Lagged Ensemble [4].

Pongamos como ejemplo el modelo HARMONIEAROME [1] de AEMET, que se ejecuta cada seis horas $(0,6,12$ y 18 UTC) con un alcance de +48 horas. Esto nos daría un total de 8 miembros para las seis primeras horas de pronóstico, 7 para las siguientes seis horas, 6 para las siguientes y así sucesivamente.

La principal desventaja para esta aproximación es que los miembros que lo componen no serían exactamente equiprobables: serían no equiprobables en una cuantía desconocida (la equiprobabilidad es un aspecto crucial en el diseño de SPC, ver sección 13.7.2 en la página 191), además del número limitado de miembros disponibles. Salvando este hecho sería razonable pensar que las pasadas anteriores a la última disponible pueden aportar información de interés para el pronóstico en caso de que dispongamos de un sólo modelo para un área espacial determinada.

Para visualizar de qué forma la información generada por pasadas anteriores puede aportar información adicional podemos tomar como ejemplo la estimación de la densidad espacial de rayos generada a partir del campo de graupel integrado en la vertical del modelo no hidrostático HARMONIE-AROME de AEMET para las 11 UTC del día 31 de julio de 2015 en el centro peninsular. La pasada más próxima a ese momento es la de las 06 UTC, y su salida se muestra en la Figura 34.2, donde se representa la densidad espacial de rayos. Vemos que se pronostica actividad eléctrica sobre la confluencia de las provincias de Zaragoza, Soria y Guadalajara y, algo menos, en las provincias de Ávila y Cuenca.

En los distintos pronósticos para esa misma hora correspondientes a pasadas anteriores, mostrados en la Figura 34.3 en la página siguiente, podemos ver que también se pronostica actividad eléctrica en zonas cercanas pero claramente no en las mismas zonas ni con la misma intensidad.

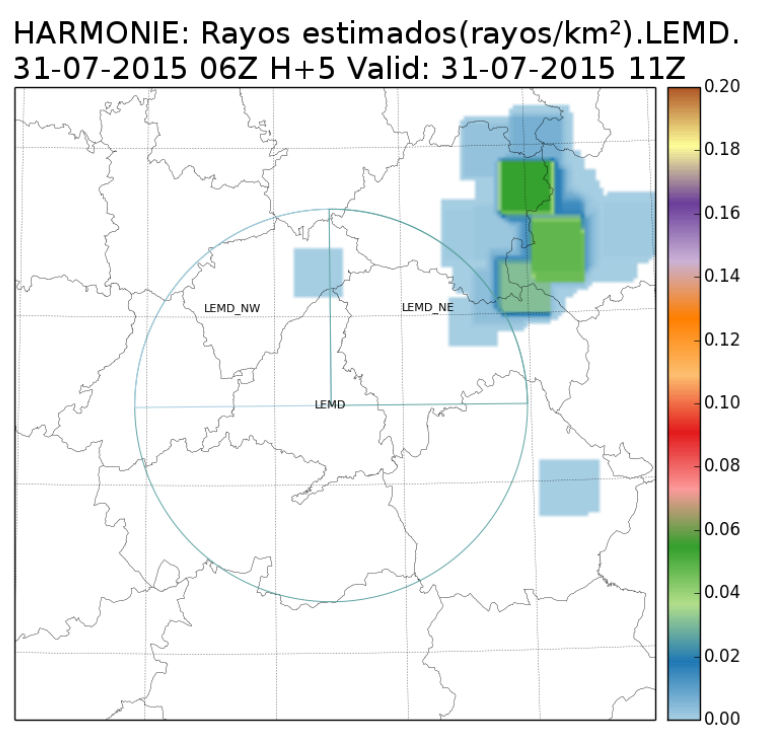

Figura 34.2: Pronóstico para las 11 UTC con la pasada de las 06 UTC de HARMONIE-AROME. 


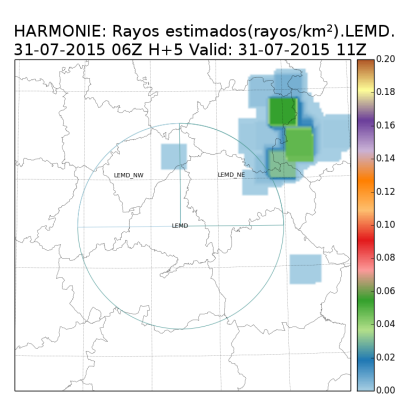

HARMONIE: Rayos estimados (rayos $\left./ \mathrm{km}^{2}\right)$.LEMD. 30-07-2015 06Z H+29 Valid: 31-07-2015 112

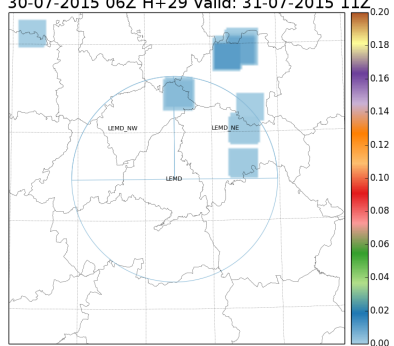

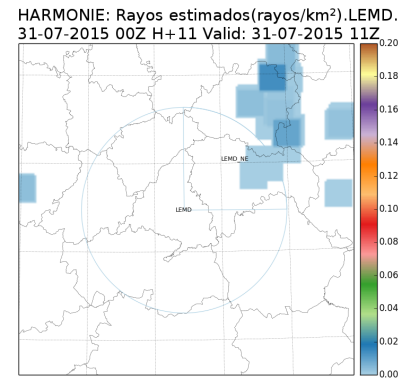

HARMONIE: Rayos estimados(rayos $\left./ \mathrm{km}^{2}\right)$.LEMD. 30-07-2015 00Z H+35 Valid: 31-07-2015 11Z

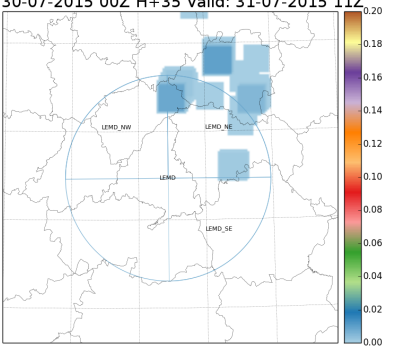

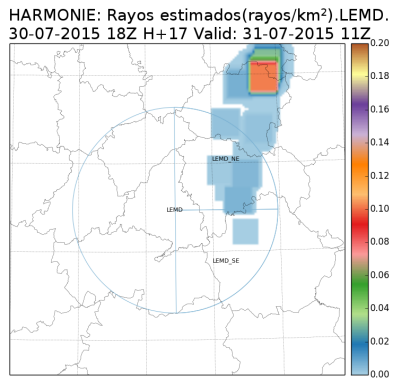

HARMONIE: Rayos estimados $\left(\right.$ rayos $\left./ \mathrm{km}^{2}\right)$.LEMD. 29-07-2015 18Z H+41 Valid: $31-07-201511 \mathrm{Z}_{0.20}$

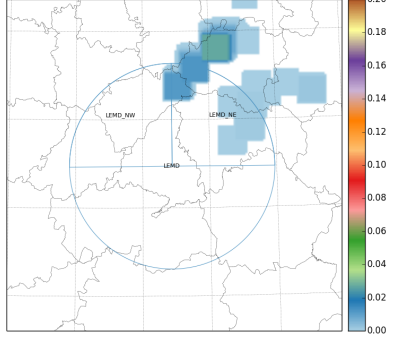

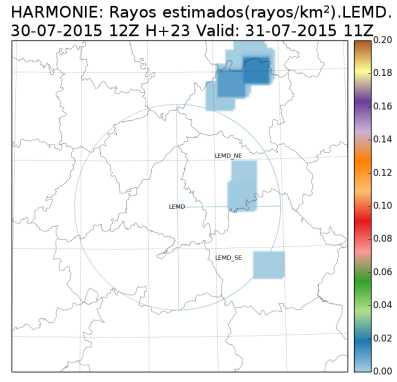

HARMONIE: Rayos estimados $\left(\right.$ rayos $\left./ \mathrm{km}^{2}\right)$.LEMD. 29-07-2015 $12 Z$ H+47 Valid: 31-07-2015 $11 \mathrm{Z}$

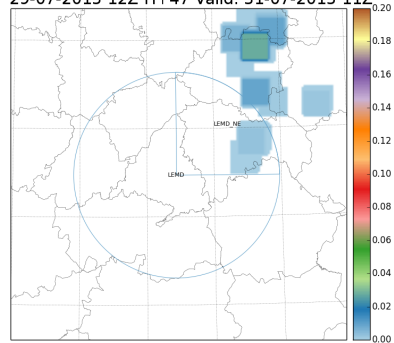

Figura 34.3: Pronóstico para las 11 UTC con la pasada de las 06 UTC y otras siete pasadas anteriores de HARMONIE-AROME.

Podemos construir un mapa que en cada punto nos represente el porcentaje de pasadas que han pronosticado algún tipo de actividad eléctrica en dicho punto, con idea de representar el grado de consenso entre las distintas pasadas, porcentaje que, con la interpretación adecuada, representa una probabilidad. Superponiendo las observaciones detectadas por la red de descarga de rayos de AEMET en puntos azules podemos comparar ambos patrones. (Figura 34.4).
Podemos observar que la rama de rayos superior que apenas era esbozada por la última pasada disponible, sí que ha sido «visualizada» por pasadas anteriores. Vemos también que la actividad esbozada por la pasada más reciente en la provincia de Cuenca al final no ha tenido reflejo en las observaciones, sin embargo la de la provincia de Ávila, que cuenta con mayor grado de consenso sí que ha sido observada.
HARMONIE: Consenso entre pasadas (\%). 31-07-2015 06Z H+5 Valid: 31-07-2015 $11 Z$

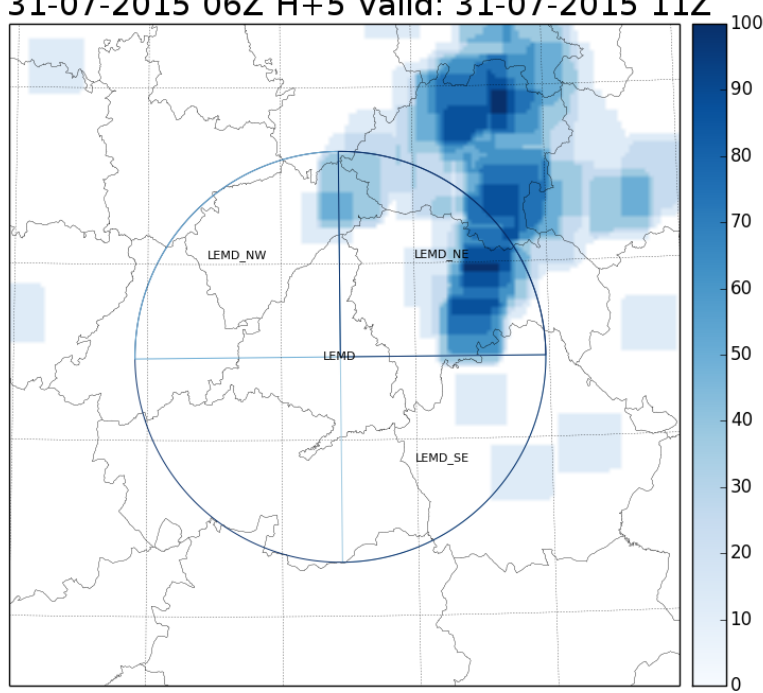

HARMONIE: Consenso entre pasadas (\%).

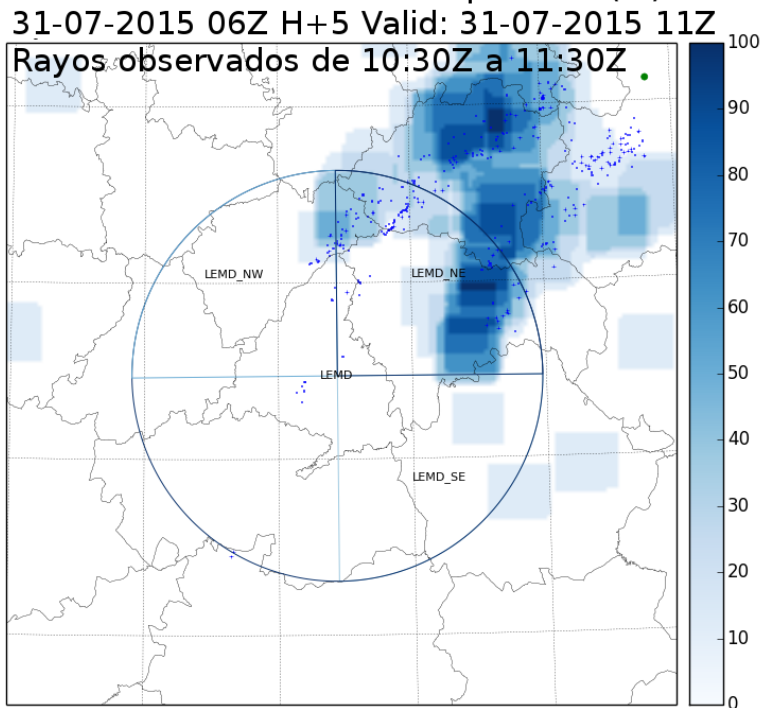

Figura 34.4: Izquierda: Pronóstico de «consenso» para las 11 UTC con las 8 pasadas citadas en la Figura 34.3 de HARMONIE-AROME. Derecha: Superposición del pronóstico de «consenso» con las correspondientes localizaciones de rayos observados (puntitos azules). 


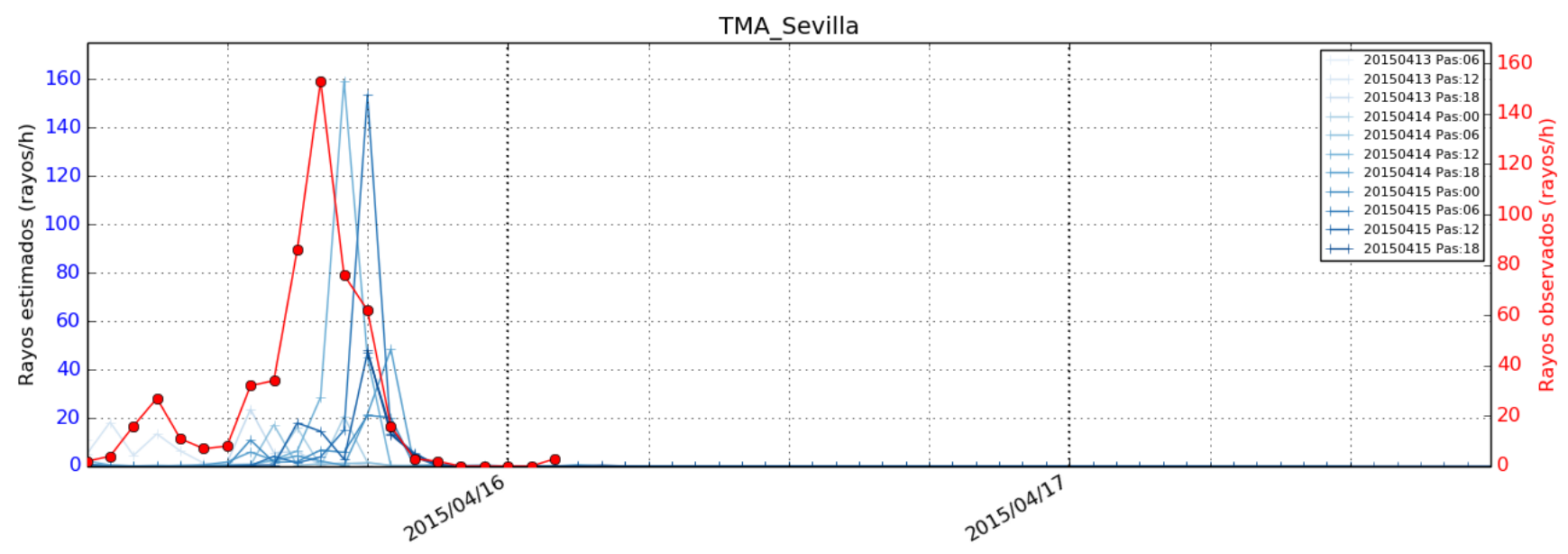

Figura 34.5: Diagrama temporal de intensidad de rayos (número de rayos por hora) en el TMA de Sevilla, comparando los estimados en azul con los observados en rojo (ver texto).

Cabría destacar también que ha habido observaciones en el interior de la provincia de Madrid, que no han sido pronosticadas por ninguna de las pasadas disponibles.

No todo lo que se modeliza llega a observarse, ni todo lo que se observa se ha modelizado adecuadamente, ni por el ensemble ni por ninguno de los miembros individuales; sin embargo, cualitativa e intuitivamente, se aprecia un mejor acuerdo con las observaciones por parte del ensemble.

También podríamos representar en un diagrama temporal la magnitud pronosticada por nuestro modelo integrada en un área espacial determinada, Figura 34.5. Se representa en los distintos tonos de azul la cantidad de rayos pronosticada por el modelo HARMONIEAROME de AEMET para el área de control de la Terminal de Sevilla (Terminal Manoeuvring Area, abreviadamente $T M A$, es un término referido en aviación al espacio aéreo controlado de gran volumen), en rojo se representan las observaciones por la red de detección de descargas de AEMET. En el eje horizontal se representa la línea temporal.

Vemos, lógicamente, que no todas las pasadas pronostican de igual forma el fenómeno observado, o mejor dicho, ninguna de las pasadas del modelo ha sido capaz de reproducir con plena exactitud lo que después se ha observado.

Sin embargo, la envolvente de todas la pasadas parece aproximarse más a lo ocurrido. $\mathrm{Y}$ es eso lo que, con un sentido crítico y realista, se pretende.

\subsection{Conclusiones}

Los modelos numéricos de predicción del tiempo producen errores que dependen del tipo de fenómeno a pronosticar, siendo la convección profunda uno de los fenómenos en los que los errores espaciales y temporales pueden ser mayores. Por otro lado, la convección puede llevar asociada actividad eléctrica.

Hemos visto cómo la variable de pronóstico granizo blando o graupel puede utilizarse para la estimación de la actividad eléctrica. Esta actividad, asociada a una de las variables pronosticadas por el modelo relacionada con la convección profunda, no es pronosticada con exactitud en el tiempo y en el espacio, o incluso puede pronosticarse y no tener lugar, o lo contrario, producirse y no haberse pronosticado. Hay que tener en cuenta también que los sistemas de observación no son perfectos y pueden darse fenómenos que éstos no hayan sido capaces de captar y viceversa. En estas circunstancias, para simular fenómenos convectivos es natural utilizar una aproximación probabilista, por ejemplo tipo ensemble, que trate de representar las incertidumbres en la modelización atmosférica.

Hemos visto también cómo todas las pasadas disponibles de un mismo modelo pueden aportarnos información sobre lo que puede ocurrir y, en primera aproximación, puede utilizarse un ensemble de hombre pobre construido utilizando estas pasadas de un mismo modelo (técnica denominada time lagged ensemble). Los miembros de un ensemble de este tipo no pueden en principio considerarse exactamente equiprobables. Dicho problema podría solucionarse 
asignando pesos a cada miembro, pesos que obviamente habría que cuantificar de forma objetiva [4]. Podemos concluir que un sistema de predicción por conjuntos de este tipo puede aportar información tremendamente útil a un coste relativamente bajo, pues es suficiente con usar las pasadas más recientes del modelo de que se disponga, en los casos presentados
HARMONIE-AROME de AEMET.

La experiencia del personal de AEMET responsable de la predicción del tiempo a corto y muy corto plazo en el uso de esta herramienta es muy positiva y, en la actualidad (2017), es una de las informaciones imprescindibles para la previsión de actividad tormentosa asociada a la convección. 


\subsection{Referencias}

[1] Bengtsson, Lisa y col. "The HARMONIEAROME Model Configuration in the ALADIN-HIRLAM NWP System". En: Monthly Weather Review 145.5 (mayo de 2017), páginas 1919-1935. ISSN: 0027-0644. DOI: $10.1175 /$ MWR-D-16-0417.1 (citado en página 565).

[2] EBERT, Elizabeth E. y EBERT, Elizabeth E. "Ability of a Poor Man's Ensemble to Predict the Probability and Distribution of Precipitation". En: Monthly Weather Review 129.10 (oct. de 2001), páginas 2461-2480. ISSN: 0027-0644. DOI: 10 . $1175 / 1520-$ 0493 (2001) 129<2461: AOAPMS>2 . 0 . CO; 2 (citado en página 565).

[3] LAfORE, Jean-Philippe y col. The MesoNH atmospheric simulation system. Scientific Documentation. Part III: Physics. Informe técnico. Météo-France, CNRS, 2009 (citado en página 564).

[4] Lu, Chungu y col. "Short-Range Numerical Weather Prediction Using Time-Lagged Ensembles". En: Weather and Forecasting 22.3 (jun. de 2007), páginas 580-595. ISSN: 08828156. DOI: $10.1175 /$ WAF999. 1 (citado en páginas 565,568$)$.
[5] MCCAUL, Eugene W. y col. "Forecasting Lightning Threat Using Cloud-Resolving Model Simulations". En: Weather and Forecasting 24.3 (jun. de 2009), páginas 709-729. ISSN: 0882-8156. DOI: 10 . 1175/ 2008WAF2222152 . 1 (citado en página 564).

[6] Poelman, Dieter R. "On the science of lightning: An overview”. En: Royal Meteorological Institute of Belgium (2010) (citado en página 564).

[7] Quiby, J y Denhard, M. "SRNWP-DWD poor-man ensemble prediction system: the PEPS project". En: Eumetnet Newsletter 8 (2003), páginas 9-12 (citado en página 565).

[8] Sosa Cardo, José Antonio, Calvo SánCHEZ, Francisco Javier y MARTín LEÓN, Francisco. "Pronóstico de rayos mediante el modelo no hidrostático HARMONIE". En: XXXIII Jornadas Científicas de la Asociación Meteorológica Española (2014). URL: http : / / hdl . handle . net / 20 . 500 . 11765/2858 (citado en página 564).

[9] WiT, Roos de y TIJM, Sander. "Prediction of lightning intensity using graupel". En: Meteorologica 19.2 (2010), páginas 21-24. URL: http : / / issuu . com/nvbm/docs / meteorologica-jun-2010 (citado en página 564). 